\title{
Capsule Commentary on Tokes et al. Disease and Treatment Perceptions Among Asian Americans Diagnosed with Chronic Hepatitis B Infection
}

\author{
Marij A. Hillen, PhD \\ Department of Medical Psychology, Academic Medical Center, University of Amsterdam, Amsterdam, The Netherlands.
}

J Gen Intern Med 29(3):517

DOI: $10.1007 / \mathrm{s} 11606-013-2697-5$

(c) Society of General Internal Medicine 2014

$\mathrm{C}$ hronic hepatitis B disproportionally affects AsianAmericans in the US. Tokes et al. ${ }^{1}$ studied AsianAmericans with chronic hepatitis $\mathrm{B}$ to evaluate their attitudes toward treatment. Using a discrete choice model, they examined how costs and potential side effects influenced patients' evaluations and decisions to (not) take medication, as well as their relative importance. They found that nearly half were reluctant to start treatment; that most subjects were aware of the potential long-term consequences of chronic hepatitis B, were aware of the treatment options, and worried most about long-term kidney damage due to medication use. Costs were a second important consideration. They also found that Asian-Americans overstated the potential impact of diet and lifestyle in controlling their disease.

The authors conclude that physician communication may play an important role in better educating these patients. This is an interesting conclusion given that they found patients had a fairly good understanding of their disease, availability of effective treatment and potential side effects thereof. Patients do, however, appear to overstate concerns about potential kidney damage and disproportionately believe in the importance of diet, lifestyle and alternative medications for managing chronic hepatitis B. This may be amenable to improved information provision. However, care should be taken that 'educating the patient' is not merely providing more information. Physicians have trouble determining their patients' beliefs, particularly with non-
Caucasian patients. ${ }^{2}$ Active patient participation in the consultation reduces this gap, and therefore needs to be enabled and stimulated. Physicians may need to deliberately yet cautiously broach sensitive issues such as patients' worries about medication costs or use of complementary medicine. Patients' illness and medication beliefs need to be explored and, when inaccurate, changed. The latter may require techniques adapted from health-behavior change methods. $^{3}$

In general, better communication has been shown to improve patient satisfaction and adherence. ${ }^{4}$ Advanced communication training can provide physicians with the necessary tools to explore patient beliefs and understanding and help them make decisions within their illness context, based on accurate information.

Conflict of interest: The author has no conflict of interest with any of the material in this article.

Corresponding Author: Marij A. Hillen, $\mathrm{PhD}$; Department of Medical Psychology, Academic Medical Center, University of Amsterdam, Amsterdam, The Netherlands (e-mail: m.a.hillen@amc.uva.nl).

\section{REFERENCES}

1. Tokes K, Guadri S, Cahill P, Chiu G, Ivanov A, Tang H. Disease and treatment perceptions among Asian Americans diagnosed with chronic hepatitis B infection. J Gen Intern Med. 2013. doi: 10.1007/s11606-013-2673-0.

2. Street RL, Haidet P. How well do doctors know their patients? Factors affecting physician understanding of patients' health beliefs. Journal of General Internal Medicine. 2011;26:21-7.

3. Elder JP, Ayala GX, Harris S. Theories and intervention approaches to health-behavior change in primary care. American Journal of Preventive Medicine. 1999;17:275-84.

4. Zolnierek KBH, DiMatteo MR. Physician communication and patient adherence to treatment a meta-analysis. Med Care. 2009;47:826-34. 